\title{
Gravitational Clustering to All Perturbative Orders
}

\author{
Elcio Abdalla ${ }^{a}$ and Roya Mohayaee ${ }^{b}$ \\ ${ }^{a}$ Instituto de Física, Universidade de São Paulo, \\ C.P. 66318, CEP 05315-970, São Paulo, Brazil \\ ${ }^{b}$ Dipartimento di Fisica Galileo Galilei (INFN) \\ Via Marzolo 8, 35131 Padova - Italy
}

Received on 8 May, 2000

\begin{abstract}
We derive the time evolution of the density contrast to all orders of perturbation theory, by solving the Einstein equation for scale-invariant fluctuations. These fluctuations are represented by an infinite series in inverse powers of the radial parameter. In addition to the standard growing modes, we find infinitely many more new growing modes for open and closed universes.
\end{abstract}

The large scale structure of the universe is believed to have grown, due to gravitational instability, from small primordial density fluctuations. These fluctuations are fully characterized by the density contrast, $\delta(t, \vec{x})=\delta \rho(t, \vec{x}) / \rho(t)$. At any given time, the connected correlation functions of the density contrast, determine the power spectrum and the space distribution of the matter in the universe $[1,2,3,4,5]$. The time dependence of the density contrast, on the other hand, determines if the inhomogeneities would indeed grow, or would just oscillate or decay, in the first place.

In this article, we are primarily concerned with the time evolution of the density contrast. In almost all standard methods, this is determined by the continuity, Euler and Poisson equations in the Newtonian regime $[1,6,7,8,9,10]$ and by the Einstein equation in the relativistic era $[1,6,7,8,9,10]$. In the nonlinear regime, the perturbation is carried to second order and new growing modes containing nonlocal terms are obtained for dust universes $[1,5,11]$. The local nonlinear terms have also been studied to all orders for the flat universe [4, 12]. A single complete treatment covering all possible cases and also extending to an arbitrary perturbative order does not exist.

Our starting point is not the usual equations of fluid hydrodynamics but the Einstein equation which naturally covers the present era too. We expand the Einstein equation around the background Friedmann universe by scale-invariant fluctuations. These fluctuations are expressed as an infinite series in inverse powers of the radial parameter. This method, which assumes spherical symmetry, allows us to obtain the full expression for the growth rate of density inhomogeneities in Newtonian, relativistic, zero and non-zero curvatures, linear and local-nonlinear regimes in a single treatment. Thus in this way, we make major simplifications on current per- turbative methods. In a flat universe, the leading growing modes are given by an infinite series in the matter and radiation -dominated eras. For the open and closed universes we find, in addition to the modes presented in the literature $[8,11]$, infinitely many more new growing modes which are also given by infinite sums. In the nonlinear regime, we also find new growing modes for closed and open universes in the extreme limits of small and large times.

Contrary to the standard analysis, where the density contrast is often taken to be a random Gaussian field, we take it to be a scale-invariant quantity. That the standard growing modes are contained in our results, is a further solid evidence that the time evolution of the density contrast is unaffected by its statistical properties.

Since, the inverse radial parameter now plays the rôle of the perturbation parameter, the order by which the structures at different scales are formed is easily determined. Our perturbation scheme implies a bottomup or a hierarchical clustering scenario for the formation of structures and thus a universe dominated by cold dark matter [8].

To account for the inhomogeneity of space-time, we use the spherically symmetric metric,

$$
\begin{array}{ll}
\operatorname{diag} \quad & \left(g_{\mu \nu}\right)= \\
& \left(-1, \frac{R_{p}^{2}(t, r)}{1-k r^{2}}, R_{p}^{2}(t, r) r^{2}, R_{p}^{2}(t, r) r^{2} \sin ^{2} \theta\right),
\end{array}
$$

where the scale factor $R_{p}(t, r)$ is a function of both time and coordinate. This metric does not assume homogeneity and is contained in the Tolman metric for a pressure-less universe [13], although not restricted to 
this era. We assume that the inhomogeneities are given by scale-invariant fluctuations and expand the scale factor as

$$
R_{p}(t, r)=R(t)+\sum_{n=0}^{\infty} \frac{\delta R_{n}(t)}{r^{n}}
$$

around the background Friedmann-Robertson-Walker scale factor, $R(t)$. This is a genuine perturbation of the metric and not just a gauge mode. A solution $\zeta_{\mu}$ of $\delta g_{\mu \nu}=\zeta_{\mu, \nu}+\zeta_{\nu, \mu}$ where $\delta g_{\mu \nu}$ is the metric perturbation, such that $\delta g_{0 r}=0$ and $\delta g_{r r} \sim t^{a} r^{-n}$, for a general $a$ and $n$, cannot be constructed [14].

The time-time and radial-radial components of the Einstein equation are expanded as in (2) and, on using the equation of state $P=\omega \rho$, are reduced to the following second-order recursive differential equation [15]:

$$
\begin{aligned}
& \left(2 \delta \ddot{R}_{l} \delta R_{s}+(3 \omega+1) \delta \dot{R}_{l} \delta \dot{R}_{s}\right) r^{-(l+s)} \\
+\quad & {\left[\left(\omega\left(3-4 n-n m+2 n^{2}\right)+n(m-2)+1\right) k\right.} \\
-\quad & \left.n(\omega(2 n-2-m)+(m-2)) r^{-2}\right] \\
& \delta R_{n} \delta R_{m} r^{-(n+m)}=0,
\end{aligned}
$$

where summations over the indices $n, m, l$ and $s$ are implied and $\delta R_{n}$ represents $R+\delta R_{0}$ when $n=0$. We have solved the above equation by a Maple program to very large orders for a flat universe, i.e. $k=0$ [16]. The solutions are substituted back in the time-time component of the Einstein equation and a series expression for the density and subsequently the leading-order growing modes for the density contrast are found. The fastest growing modes can be written in the closed forms

$$
\delta_{+} \sim \sum_{n=0}^{\infty} C_{2 n+3} \frac{t^{(2 n+2) / 3}}{r^{2 n+3}},
$$

in the matter-dominated era, $\omega=0$, and

$$
\delta_{+} \sim \sum_{n=0}^{\infty} C^{\prime}{ }_{2 n+3} \frac{t^{n+1}}{r^{2 n+3}}
$$

in the radiation-dominated era. We see that in addition to the usual growing modes, $t^{2 / 3}$ and $t$ in the matter and radiation dominated eras respectively, we have infinitely many more growing modes. The higher growing modes are the local nonlinear modes $[1,12]$. The above density contrasts also tell us that smaller structures enter nonlinear regime, $\delta>1$, faster than the larger structures. Thus a hierarchical clustering scenario is expected in this scheme.

For the closed and open universes, the recursive equation (3) is highly inhomogeneous and cannot be solved. However, assuming that different perturbative orders evolve independently of each other, we find, in the matter-dominated era, the linearized equation

$$
\frac{d^{2} \delta R_{n}}{d \psi^{2}}+\left(n-\frac{1}{2 \sinh ^{2} \frac{\psi}{2}}\right) \delta R_{n}=0
$$

where $\psi$ is real for an open and is imaginary, $\psi=-i \theta$, for a closed universe. The above equation can be solved exactly. The solutions can be used in the Einstein equation to obtain the density. The growing modes of the density contrast are given by

$$
\begin{aligned}
\delta_{+}(\psi) & \sim \frac{\sin (\sqrt{n} \psi)}{\sqrt{n}}\left[\frac{3 \sinh \psi}{(1-\cosh \psi)^{2}}+\frac{2 n \sinh \psi}{1-\cosh \psi}\right] \\
& +\cos (\sqrt{n} \psi)\left[\frac{5+\cosh \psi}{1-\cosh \psi}\right] .
\end{aligned}
$$

We see that in addition to the usual growing mode [8] which is given by $n=0$ terms in the above equation, infinitely many more growing modes exist in the linear regime. The $n \neq 0$ modes have been overlooked in the previous analyses and can have important consequences for the power spectrum.

In the nonlinear regime, the full inhomogeneous equation (3) for open and closed universes can also be solved analytically at low orders and by a fully algebraic Maple program at higher orders [15] in the limits of small and large times. At small times, the first growing modes $\delta_{+} \sim t^{2 / 3}$ and $\delta_{+} \sim t$ in the matter and radiation-dominated eras occur at the lowest order in the perturbation series, i.e. at the r-independent order [15]. At higher orders the solution contains oscillatory and or decaying modes only. Therefore, open and closed universes behave as a flat universe at small times in the linear regime, as expected. However, this statement is not completely true. In a flat universe the first growing modes appear at the third, $r^{-3}$, rather than the first, order in the perturbation expansion, as expressed by equations (4) and (5). In other words, at small times the inhomogeneities will enter the nonlinear phase faster in the open and closed universes than in the flat universe. Therefore, unlike common expectations, structures can grow faster in an open universe than in a flat universe.

At large times, a new growing mode arises in an open universe. Taking the asymptotic limit of the hypergeometric solutions to the Einstein equation [15] we obtain

$$
\delta_{+} \sim \frac{t^{3 \omega / 2}}{r}
$$

This result indicates that, at very large times, inhomogeneities will only grow in an open universe if the radiation pressure is non-vanishing. However, since the baryonic matter cannot grow in the radiation-dominated era due to its strong coupling to the radiation, the above mode is only relevant for the growth of the perturbation in the non-baryonic dark matter component.

We, therefore, conclude that for an open universe, in addition to the growing solution appearing at the zero mode (i.e. at $n=0$ in equation $(7)$ ), many more 
growing modes exist. These modes have not been obtained previously, even though the flat universe modes, (4) and (5), have been well known in the context of the relativistic theory of small perturbations and spherical top hat model (see for example [8]). Our method of finding the evolution of a specific type of perturbation, namely a scale-invariant fluctuation, enables us to obtain the expression (3), which can be solved in general without being restricted to an Einstein-de Sitter dust universe in a linear regime. The extra growing modes presented in equation (7) can have significant consequences for the formation of structure in an open universe. It would also be interesting to obtain the nonlinear-nonlocal growing modes [1]. We shall discuss both these issues in future works.

E.A thanks Conselho Nacional de Desenvolvimento Científico e Tecnológico (CNPq-Brazil) and Fundação de Amparo a Pesquisa do Estado de São Paulo (FAPESP) for financial support.

\section{References}

[1] P.J.E. Peebles, The large scale structure of universe (Princeton University Press, NJ, 1980).

[2] J.N. Fry, Astrophys. J. 279, 499 (1984).

[3] M.H. Goroff, B. Grinstein, S.-J. Rey and M.B. Wise, Astrophys. J. 311, 6 (1986).

[4] R. Scoccimarro, S. Colombi, J.N. Fry, J.A. Frieman, E. Hivon, A. Melott, Astrophys. J. 496, 586 (1998); R. Scoccimarro and J. Frieman, Astrophys. J. 473, 620 (1996); R. Scoccimarro and J. Frieman, Astrophys. J. Supp. 105, 37 (1996).
[5] F.R. Bouchet, S. Colombi, E. Hivon and R. Juszkiewicz, Astronom.\& Astrophys. 296, 575 (1995).

[6] S. Weinberg, Gravitation and Cosmology (John Wiley and Sons, NY, 1972).

[7] E.W. Kolb and M.S. Turner, The early Universe (Addison Wesley, Redaing, Mass., 1990).

[8] P. Coles and F. Lucchin, Cosmology: The origin and evolution of cosmic structure (John Wiley \& Sons Ltd, West Sussex, 1995).

[9] V. Sahni and P. Coles, Phys. Rep. 262, 1 (1995).

[10] F.R. Bouchet, Lecture notes for the Course CXXXII of the Enrico Fermi School on Dark Matter in the Universe (Varenna, 1996); Report No. astro-ph/9603013.

[11] H. Martel and W. Freudling, Astrophys. J. 371, 1 (1991).

[12] P. Fosalba and E. Gaztañaga, Report No. astroph/9712095, Report No. astro-ph/9712263.

[13] G. Lemaître, Ann. Soc. Sci. Bruxelles A53, 51 (1933); G. Lemaitre, Gen. Rel. Grav. 29, 641 (1997); R.C. Tolman, Proc. Natl. Acad. Sci. 20, 169 (1934); H. Bondi, Mon. Not. R. Astron. Soc. 107, 410 (1947).

[14] G.F.R. Ellis and M. Bruni, Phys. Rev. D 40, 1804 (1989).

[15] E. Abdalla and R. Mohayaee, Report No. astroph/9810146, Report No. astro-ph/9711162.

[16] The complete algebraic Maple programs can be found at the site http://www.fma.if.usp.br/ ${ }^{\sim}$ fractals/perturbation. 